\title{
Rotational Micromanometers
}

\author{
Karl Lofquist
}

\author{
(June 7, 1962)
}

\begin{abstract}
A rotational manometer may be defined as an instrument which measures a pressure difference by balancing against it a known pressure difference generated by a rotating element. A single fluid is used, and balance is determined by the absence of flow in a detector. In one type, the centrifugal manometer, the rotor-generated pressure is independent of molecular viscosity and is predictable. This instrument, which has been previously investigated and tested in air, is here adapted for use in water. Under the conditions of test, it proved repeatable to within about \pm 2 percent, for pressure differences above 1 dyne $\mathrm{cm}^{-2}$, and to about \pm 10 percent for pressure differences down to $1 / 10$ dyne $\mathrm{cm}^{-2}$. An alternate type, in which the rotor-generated pressures depend upon viscosity, was found, when tested, to be unsatisfactory. A simple general analysis, supported by observations, shows the dependence of manometer sensitivity upon the resistance of the system and the design of the flow detector.
\end{abstract}

\section{Introduction}

Kemp ${ }^{1}$ has described a micromanometer in which a known pressure difference is generated by centrifugal accelerations in a rotor to balance an unknown pressure difference. Balance is determined by the absence of flow in a detector containing suspended particles. He has constructed such an instrument, the "centrifugal manometer," for use in air and has found it to perform very satisfactorily.

A manometer if this type has several advantages for measurement of a pressure difference between two points in a given fluid, providing only that the difference is nearly steady. It contains but a single fluid, the same as that given. Consequently, with no fluid in contact with another, there is no meniscus or bubble and no column of liquid to be measured. Any change in the unknown pressure difference accelerates the flow in the detector almost immediately, and there is no lag associated with the filling and emptying of reservoirs. This advantage is much greater for measurements in water than in air, as such a lag increases with the inertia and viscosity of the fluid. Since the operation of the manometer is independent of the action of gravity, it does not need to be level and does not require a steady platform. The principle of operation is simple and, with careful design, the performance can be predicted with accuracy sufficient to make a calibration unnecessary. Finally, since the known pressure difference created within the instrument does not require thermal expansion or compressibility of the fluid, such a manometer is suitable for use in a liquid.

The primary effort described here is the design and test of a centrifugal manometer adapted for use in water. This adaptation involves, on the one hand, such practical problems as the choice of particles to be suspended and the control of bearing leakage, bubble formation, and temperature varia-

1 J. F. Kemp, Centrifugal manometer, J. Basic Eng., pp. 341-347 (Sept, 1959). tions. On the other hand is the question of to what extent the predictability of performance, found by Kemp, will be repeated in a pressure generator of quite different size and design, in particular, with a rotor of much smaller diameter.

Secondly, as will be seen, the concept of the centrifugal manometer is easily generalized to that of the "rotational" manometer, a class which includes many additional possible designs. Since these designs differ only in their pressure generators, retaining the same system of rotor drive and flow detection, it was found convenient to test one and compare its performance with that of the centrifugal manometer.

Finally, the sensitivity of manometers of a rotational type is investigated to determine upon what factors it depends and how it can be increased.

\section{General Description}

\subsection{Principle of Rotational Manometers}

The general principle of the rotational manometer is easily derived. It will be found most convenient to treat the centrifugal manometer and the alternative "viscous" manometer later as special cases.

If a solid of revolution is rotated about its axis in a cavity filled with a fluid, the pressure varies from point to point throughout the fluid. Points within the solid may be included if they are accessible through suitable passageways. It is assumed that the rate of rotation is constant and that the pressure at all points is steady. Denoting the pressure difference between any two selected fixed points by $\Delta p$, a characteristic length of the system by $L$, the density and kinematic viscosity of the (incompressible) fluid by $\rho$ and $\nu$, and the rate of rotation by $\Omega$, a simple dimensional analysis shows that

$$
L^{2} \frac{\Delta p}{\rho \nu^{2}}=f\left(\frac{L^{2} \Omega}{\nu}\right)
$$


or alternately,

$$
\frac{\Delta p}{L^{2} \rho \Omega^{2}}=F\left(\frac{L^{2} \Omega}{\nu}\right)
$$

The function $f$, or $F$, can be determined by experiment and, in exceptional cases, by theoretical analysis.

If now, in a separate fluid of the same properties, a pressure difference $\Delta p^{\prime}$ exists between two given points (as say on a pitot-static tube) and these points are connected by suitable conduits to the previously mentioned points in the cavity, a current will, in general, flow through the conduits. But if the rate of rotation is adjusted such that $\Delta p$ equals $\Delta p^{\prime}$, the current will stop. Thus, if $f$ is known and $\Delta p^{\prime}$ is unknown, the cavity and rotating solid, with a motor and drive to rotate it, and with a sensing element to detect conduit flow, constitute a rotational manometer which determines $\Delta p^{\prime}$ by eq (1) whenever the flow is stopped. Conversely, if $\Delta p^{\prime}$ can be given known values, the same apparatus can be used to determine $f$.

\section{Water Manometer System}

The components and general scheme of the water rotational manometer are shown in the photograph in figure 1 . The motor and drive at the right turn the rotor within the cavity housing seen at the left center. The pressure generator shown is that of the centrifugal manometer. The flow detector is at the far left. In operation, the conduit at the left of the three open nipples shown in front of the cavity-rotor unit is connected to the external source of lower pressure. It then passes through the detector and into the rotor or cavity. The circuit is completed by the conduit in the center which connects the cavity to the external source of higher pressure.

It is necessary to prevent any leak or bubble formation in the system as these cause spurious movement through the flow detector. The conduit at the right in figure 1, which is not part of the circuit, connects an external water source to a point near the outside end of the drive shaft bearing to make the pressure at this point nearly equal to that in the cavity. Any leak out of the bearing, when this

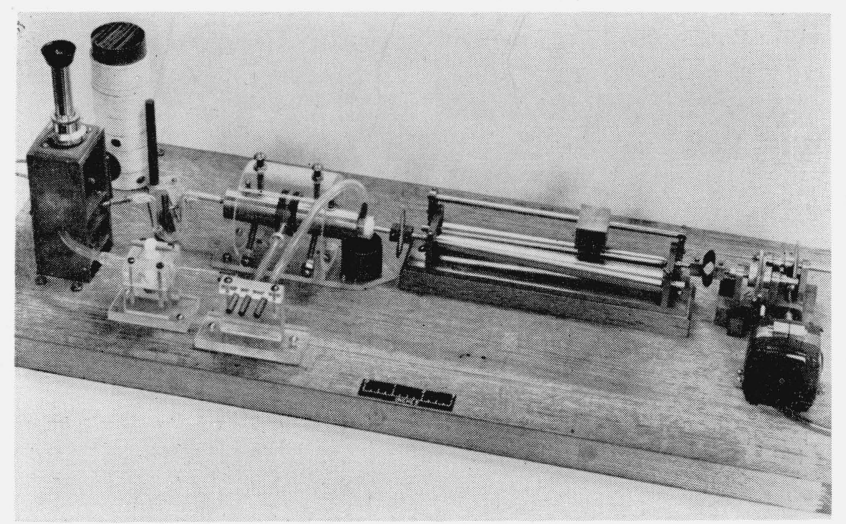

Figure 1. The water rotational manometer. pressure is greater than atmospheric, is then drawn from the external source only. If the cavity pressure is less than atmospheric, the bearing conduit can be used to keep air from entering the system. This method of leak prevention, rather than the use of some form of packing gland, was chosen to keep the drive shaft bearing friction negligible compared with the friction between the cones in the drive system described below. To prevent bubble formation, water used to fill the system was previously degassed by boiling. Since the volume flow during an observation was extremely small, this water remained in the system practically indefinitely.

In any rotational manometer it is necessary that the rate of rotation can be given any value within the operating range and there be held constant. For these purposes, a synchronous motor was used with a drive which included a system of gear selections and a pair of slightly tapered cones for continuous speed control. Torque was transmitted from one cone to the other by a weighted wheel with a narrow rubber (O-ring) perimeter. These components can be seen in figure 1.

In the calibrations, the period $T=2 \pi / \Omega$, rather than $\Omega$, was measured directly. This was done by timing, with a stop watch, a convenient number of cycles of the rotor shaft, the number chosen so that the timing lasted for around $1 \mathrm{~min}$. The readings were found to be repeatable to within about $0.3 \mathrm{sec}$, that is, to about $1 / 2$ percent, except at the highest speeds when some slip between the cones became noticeable. In the calibrations, values of $T$ ranged from $14.0 \mathrm{sec}$ down to 0.35 sec.

The flow detector was similar to that described in footnote [1], except that there the suspended particles were oil droplets. A narrow shaft of light from a vertical filament passed through a glass-walled section of the conduit and illuminated small particles in the water. Light rays emanating from the particles were then deflected upward by a prism and observed
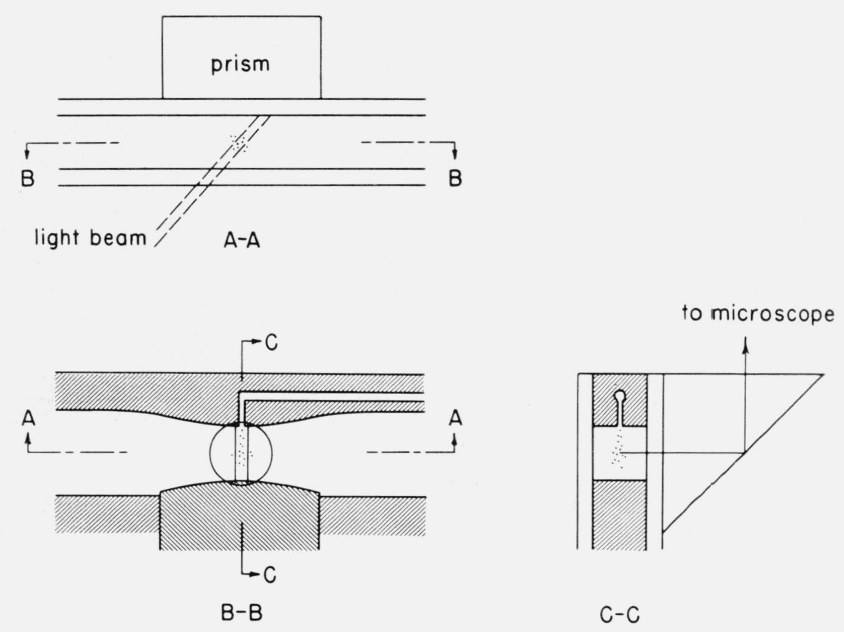

Figure 2. The design of the flow detector in the vicinity of the suspended particles.

(The glass walls are 1/16-in. apart.) 
through a low power (about $70 \times$ ) microscope. The angle between the shaft of light and the normal to the walls was sufficient (about $45^{\circ}$ ) to provide a dark background for the illuminated particles. These were of fine clay which, it was found, usually contained miscellaneous organic material. The particles could be replenished through a hole in the top of the section from a syringe serving as a reservoir. The smaller particles, whether clay or organic (it was seldom obvious), showed no tendency to settle and the reservoir was needed only occasionally.

The design of the flow detector in the vicinity of the suspended particles is shown schematically in figure 2. The circle, drawn on the side view, shows the approximate field of vision, seen through the microscope, and the two vertical lines within it represent thin wires which were placed in the focal plane within the barrel of the microscope.

\section{Calibration Systems}

Known pressure differences were provided by two calibration systems. The first consisted of two bottles of known diameters and partly filled with water, with a syringe, connected to a micrometer which injected a known volume of water into one of the bottles, thereby increasing the depth of water by a known amount. The useful range in $\Delta p^{\prime}$ for the bottles was, roughly, between 100 dynes $\mathrm{cm}^{-2}$ and 1 dyne $\mathrm{cm}^{-2}$. In a second system, the "Poiseuille apparatus," the pressure difference between two taps on the side of a long circular pipe, with carefully measured diameter, was calculated from the known rate of flow. This flow, from one vessel to another, passing through the pipe and any one of several capillaries, was previously determined in terms of the kinematic viscosity $\nu$ and the differences in water level in the vessels. These differences were substantial, between 5 and $50 \mathrm{~cm}$. This apparatus could provide any desired value of $\Delta p^{\prime}$ less than about 20 dynes $\mathrm{cm}^{-2}$. In figures 4 and 5 , the calibrations with each system are differentiated.

The probably error in the pressure difference calculated with the Poiseuille apparatus can be estimated in terms of errors in the separate measurements upon which the calculation depends. With the diameter of the pipe and the distance between pressure taps given by $d$ and $l$, and with the molecular viscosity of the liquid and the volume rate of flow given by $\mu$ and $q$, the expression for the pressure difference

$$
\Delta p==\frac{8}{\pi}\left(\frac{2}{d}\right)^{4} l_{\mu q}
$$

shows that

$$
\frac{\delta \Delta p}{\Delta p}=-4 \frac{\delta d}{d}+\frac{\delta l}{l}+\frac{\delta \mu}{\mu}+\frac{\delta q}{q}
$$

where $\delta$ denotes an error in measurement. The diameter of the section of the pipe containing the taps was determined with a slightly tapered plug. Of over a dozen readings, none deviated from the mean (0.3031 in.) by more than 0.10 percent which is taken as $\left|\frac{\delta d}{d}\right|$. The error $\delta l$ is taken to be the diameter of the pressure taps $(0.74 \mathrm{~mm})$ so that, with $l=10.0 \mathrm{~cm},\left|\frac{\delta l}{l}\right|=0.74$ percent. The error in viscosity is proportional to an error in temperature taken to be $0.1{ }^{\circ} \mathrm{C}$, the smallest division on the thermometer. At room temperature $\left(25^{\circ} \mathrm{C}\right)$ this gives $\left|\frac{\delta \mu}{\mu}\right|=0.23$ percent. Finally, in the calibration of the Poiseuille apparatus, the difference in water level in the vessels was plotted as a function of time. The derivatives of these curves, taken at numerous points, were than plotted to give $q$, the volume rate of flow. On the basis of the scatter in these plots, $\left|\frac{\delta q}{q}\right|$ is taken as 0.5 percent. Taking as the probable error in $\Delta p$ the square root of the sum of the squares of the terms on the right side of eq (3), it is found that

$$
\left(\frac{\delta \Delta p}{\Delta p}\right)_{\text {prob }}=1.10 \%
$$

If the fixed error due to the first two terms is disregarded, the variable error due to the last two terms is found to be 0.55 percent, which is a measure of the degree of scatter in the manometer calibrations attributable to the Poiseuille apparatus.

The accuracy of the bottle apparatus is difficult, to estimate since it is limited by the regularity of the shape of the menisci in the bottles and by the steadiness of the supporting platform. However, that the probable percentage error in the bottle apparatus in its useful range is at least no larger than that of the Poiseuille apparatus may be inferred from a comparison of the degree of scatter associated with each instrument in the region where they overlap, as found in the calibration in figure 4.

\section{Centrifugal Manometer}

The principal features of the pressure generator of the centrifugal manometer tested here are shown in figure 3. The cavity and rotating solid were concentric circular cylinders, separated by a constant gap, small compared with their length. The difference in pressure was taken between the inner wall of the cavity and the axis which were connected by 16 passages arranged in two rings. The axial pressure was transmitted outward through the hollow bearing to the left, while the pressure at the cavity wall was transmitted through four passages to a collecting ring. The hollow bearing at the left of the diagram and the drive shaft, which was soldered into the rotor, were of stainless steel; all other parts were of brass.

The diameters of the cavity and rotor were 1.746 and $1.581 \mathrm{~cm}$ (compared with $22.8 \mathrm{~cm}$ in Kemp's 


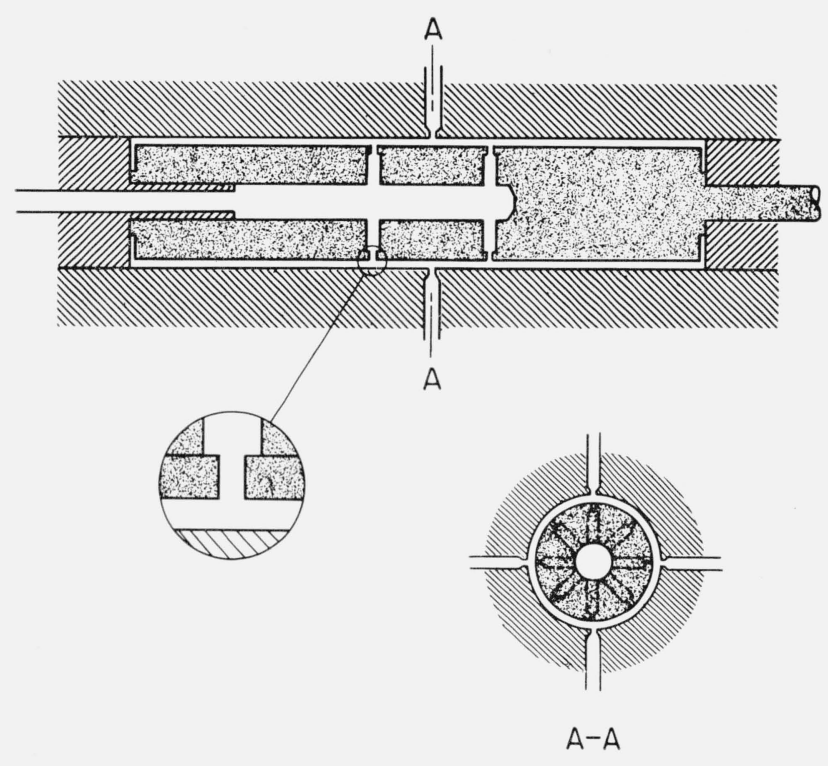

Figure 3. Design of the cavity and rotor of the centrifugal manometer.

(The diameter of the cavity is $1.75 \mathrm{~cm}$.)

manometer) leaving a gap of $0.825 \mathrm{~mm}$, about onetenth of the radius of the rotor. Their lengths were $7.91 \mathrm{~cm}$ and $7.80 \mathrm{~cm}$, almost one hundred times the gap between them, and leaving at each end a gap of $0.55 \mathrm{~mm}$. Orifices in the rotor were of $0.74 \mathrm{~mm}$ diam. for a length of $1.20 \mathrm{~mm}$ thereafter increasing to 1.60 $\mathrm{mm}$ diam. Those in the cavity walls were of approximately the same design. The diameter of the bearings was $0.476 \mathrm{~cm}(3 / 16 \mathrm{in}$.$) , and the inner$ diameter of the hollow bearing was $0.325 \mathrm{~cm}$.

As shown by the analysis in footnote 1, for a centrifugal manometer long enough to ignore end effects, and with laminar flow in the annular cavity, eq (2) takes the simple form:

$$
\frac{\Delta p}{L^{2} \rho \Omega^{2}}=K, \text { a constant. }
$$

In this case the pressure difference is unaffected by the viscosity. Taking as $L$ the radius of the rotor, the analysis gives $K$ as a function of the ratio of the gap between cylinders to the radius. As the gap is reduced to zero, $K$ becomes equal to $1 / 2$. For the manometer here described, $K$ takes the value 0.533 . However the pressure along the rotor axis may differ from that within the connecting bearing conduit where rotation of the fluid does not penetrate. Assuming that the average pressure within the inner radius of the bearing conduit is the same in the rotor as in the conduit and substituting this average for the axial pressure, $K$ is reduced to 0.5224 . Introducing $T=2 \pi / \Omega$ and the numerical value of $L$ in $\mathrm{cm}$, and with $K=0.5224$, the predicted calibration is

$$
\frac{\Delta p}{\rho} T^{2}=12.89 \mathrm{~cm}^{2} .
$$

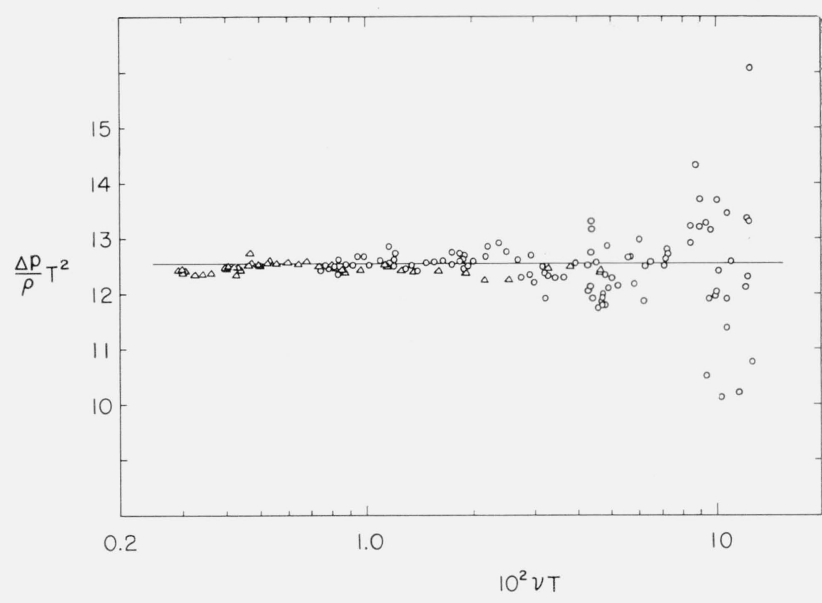

Figure 4. Calibration of the centrifugal manometer, pressure coefficient against the period of rotation.

The symbols $\Delta$ and $\bigcirc$ refer to calibration with the bottles and the Poiseuille apparatus respectively.

Figure 4 shows the calibration of the centrifugal manometer obtained by test and plotted according to eq (2). The abscissa, $10^{2} \nu T$, is a substitute for $L^{2} \Omega / \nu$. A constant value of the ordinate, of 12.55 $\mathrm{cm}^{2}$, provides about the best fit for the data. This value is about $2 \frac{1}{2}$ percent less than that predicted by eq (4). To see more readily what pressure differences the observations represent, the data have been replotted in figure 5 according to eq (1). Since for water at room temperature, $10^{2} \nu \approx 0.9$ or, roughly, unity, the ordinate in figure 5 approximates the measured pressure difference in dynes $\mathrm{cm}^{-2}$. Similarly, the abscissa in figures 4 and 5 approximates the period in seconds. The figures show that, under the conditions of the calibration, the manometer readings had a scatter of less than about \pm 2 percent for $\Delta p>1$ dyne $\mathrm{cm}^{-2}$ and of about \pm 10 percent for $\Delta p$ around $1 / 10$ dyne $\mathrm{cm}^{-2}$. As $\Delta p$ is further reduced, the scatter increases rapidly.

The deviation of the experimental calibration from the theoretical prediction is roughly of the same magnitude as the correction in $K$ from 0.533 to 0.5224 which was based upon an assumption. A reduction in the inner radius of the bearing conduit reduces both the correction and the theoretical uncertainty and, it may be assumed, would also reduce the discrepancy between the observed and predicted calibrations. In Kemp's design, where the ratio of the radius of the bearing conduit to the radius of the rotor was about $\frac{1}{6}$ of that here, no such discrepancy was apparent.

An investigation made by G. I. Taylor ${ }^{2}$ shows that the simple laminar flow in the annulus of the present manometer breaks down when $10^{2} \nu T$ is reduced to about 0.166 . This value, corresponding to $10^{-4} \frac{\Delta \rho}{\rho \nu^{2}} \approx 457$, is beyond the range of the calibra-

${ }^{2}$ H. Schlichting, Boundary Layer Theory, 1st English ed., p. 359 (McGraw Hill Book Co., New York, 1955) 


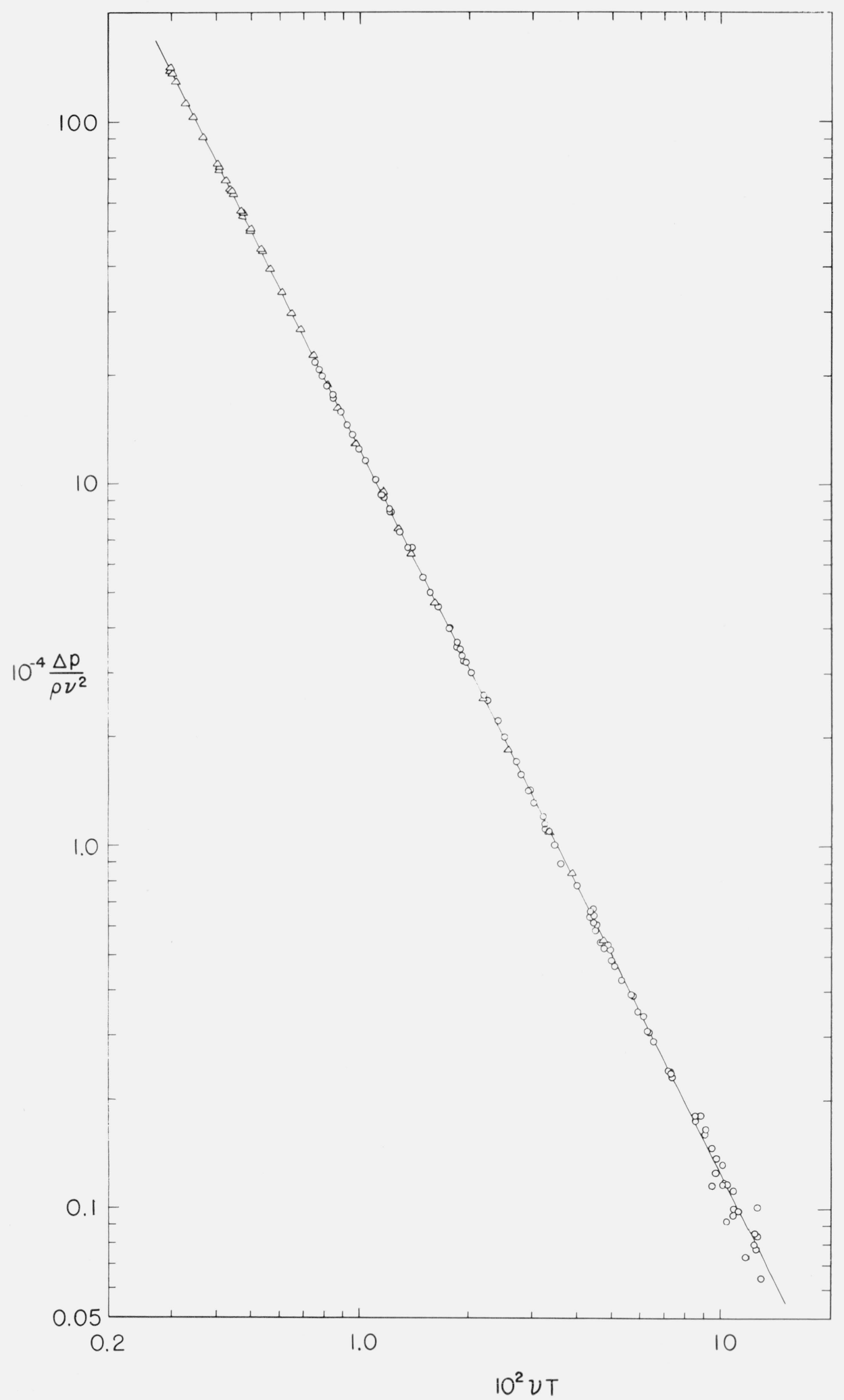

FiguRE 5. Calibration of the centrifugal manometer, pressure difference against the period of rotation. The symbols $\triangle$ and $\bigcirc$ refer to calibration with the bottles and the Poiseuille apparatus respectively. 
tions. Presumably, however, the change of regime would have little effect on the calibration curve, since Kemp's data, which contain the point of instability within their range, show no such effect.

There is no apparent upper limit to the straight line calibration curve in figure 5 . However, at the higher rates of rotation a slipping of the drive between the cones began to appear. Thus an upward extension of range would require an improvement of the drive system.

The flow through the test section was found to respond to any change in rotor speed without appreciable lag. This is because the liquid in the radial passages of the rotor moves almost as a rigid body, and its angular acceleration does not depend upon viscous action.

\section{Viscous Manometer}

In types of rotational manometers other than the centrifugal manometer the pressure difference depends in general, upon the viscosity. This is a practical disadvantage. Conceivably, however, some designs may be of practical interest because of the possibility of extreme simplicity in the configuration of cavity and rotor.

The design of the pressure generator of the "viscous" manometer tested here is shown in figure 6. The cavity and rotor are circular cylinders with axes parallel but eccentric producing a varying gap and pressure along the perimeter. The points of pressure difference are both on the inner surface of the cavity at opposite ends of a diameter. Two configurations were examined. In one, type A, the pressure taps are at $\theta= \pm \frac{\pi}{2}$, and in the other,
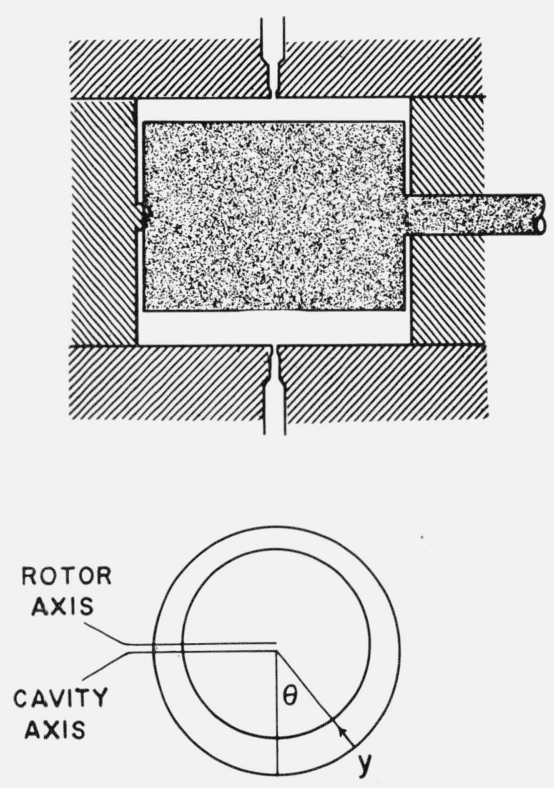

Figure 6. Design of the cavity and rotor of the viscous manometer.

(The diameter of the cavity is $15 \%$ in.) type $\mathrm{B}$ the taps are at $\theta=0, \pi$. The design is very simple with no conduits in the rotor. All parts were made of brass except the shaft which was of stainless steel. The temperature was taken in a brass well fitted along the outside of the cavity casing.

The diameters of the eavity and rotor were $1 \frac{5 \%}{8}$ in. and $1 \frac{11}{4} \mathrm{in}$. The rotor axis was displaced $3 / 64$ in. from that of the cavity so that the gap between cylinders varied from $\% / 6$ to $15 / 64$ in. The lengths of the cavity and rotor were $1^{13} / 16$ and $1 \frac{3 / 4}{4}$. leaving a gap of $1 / 32$ in. at each end. The design of the orifices in the cavity wall was similar to that already described. The shaft and bearing were $1 / 4 \mathrm{in}$. in diameter.

The principle of operation is simple. Denoting the distance and velocity along a streamline by $s$ and $u$, the equation of motion for flow in the annulus can be written, approximately,

$$
\mu \frac{\partial^{2} u}{\partial y^{2}}=\frac{\partial p}{\partial s}+\rho \frac{d u}{d t}=\frac{\partial}{\partial s}\left(p+\rho \frac{u^{2}}{2}\right)
$$

The term on the left is the resultant force per unit volume along $s$ due to viscous action and is positive when the velocity profile is concave in the direction of flow. Because of continuity of flow through the annulus, the profiles are concave for $-\frac{\pi}{2} \leq \theta \leq \frac{\pi}{2}$, approximately, and convex otherwise. Denoting. by $\bar{p}$ and $\overline{u^{2}}$ the averages between the rotor and the cavity wall for given $\theta, \bar{p}+\rho \frac{\overline{u^{2}}}{2}$ is a maximum near $\theta=\frac{\pi}{2}$ and a minimum near $\theta=-\frac{\pi}{2}$. And since at these two points the values of $\overline{u^{2}}$ may be assumed equal, there is a difference in pressure between them which is proportional to the molecular viscosity. On the other hand, $\bar{p}+\rho \frac{\overline{u^{2}}}{2}$ has about the same value at $\theta=0$ and $\theta=\pi$ and since, by continuity, $\overline{u^{2}}$ is not the same, there is a difference in pressure between these two points approximately equal to the difference in $\rho \frac{\overline{u^{2}}}{2}$.

The gap and eccentricity were made sufficiently large to insure that imperfections of construction would be of no consequence, but otherwise their dimensions were selected to produce a range of pressure about the same as that of the centrifugal manometer for the same range of rotationalspeeds. This selection was based upon an analysis of the pressure variation around the cavity wall. Because of its approximate nature, this analysis could hardly be very accurate and it is not included here. However the calibrations thus predicted for types A 


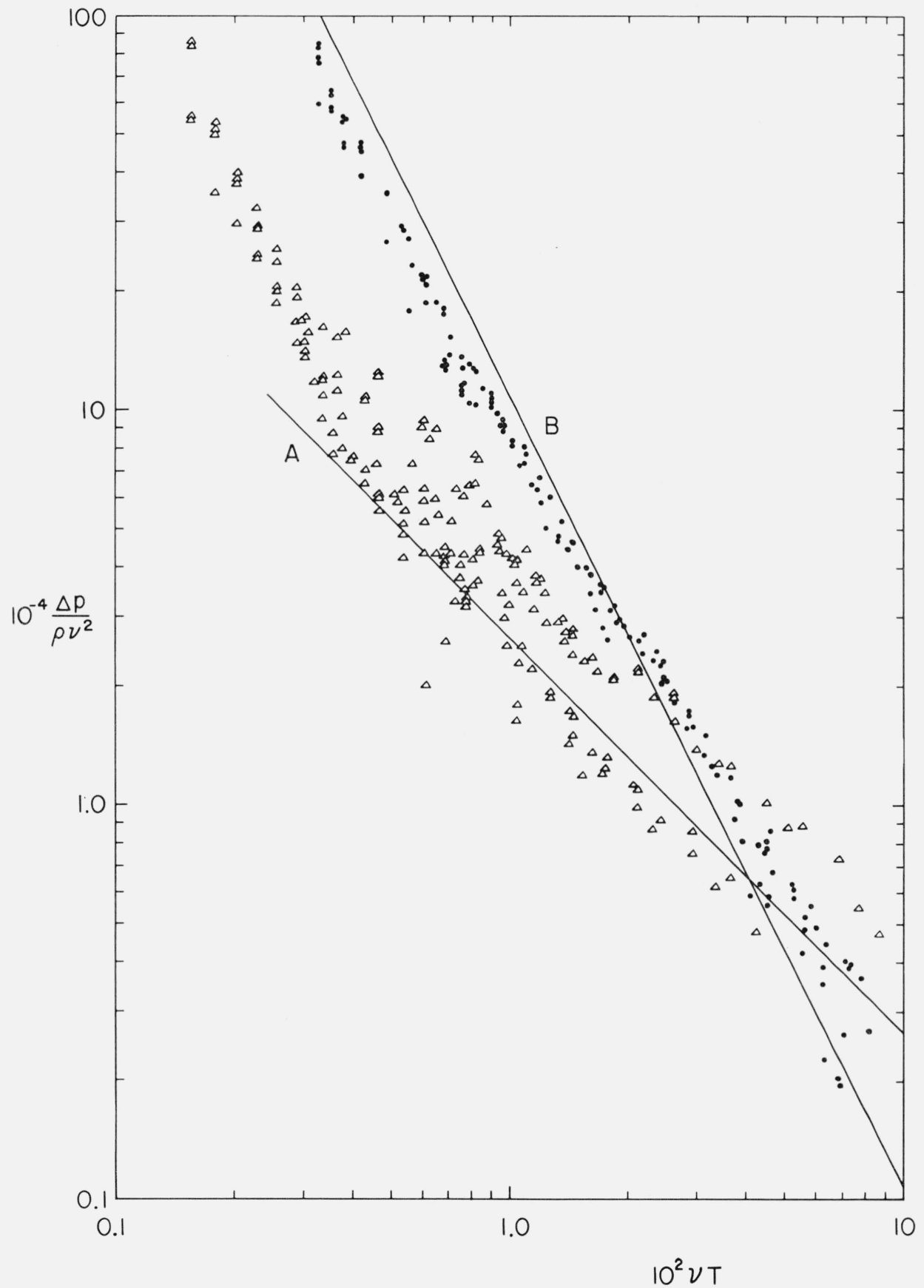

Figure 7. Calibration of the viscous manometer, types $A$ and $B$, pressure difference against the period of rotation.

The symbols $\triangle$ and $\bullet$ refer to types $\mathrm{A}$ and $\mathrm{B}$ respectively.

and $B$ are shown in figure 7 . In the first case the pressure difference is proportional to $\mu \Omega$ while in the second it is proportional to $\rho \Omega^{2}$.

The outstanding feature of the calibration data plotted in figure 7 is the high degree of scatter compared with that of figures 4 and 5 . The proven reliability of the sensing apparatus in calibrating the centrifugal manometer shows that, primarily, this scatter is not a random error in the observation of $\triangle p$, but rather is due to variations in $\Delta p$ itself. That is, the initial assumption of steady pressure is violated. Indeed, for a given $\nu T$, an observed value of $\triangle p / \rho \nu^{2}$ was frequently repeated several times consecutively, with small variation, and then observed to shift to a new value. A nonrandom variability in $\triangle p$ is apparent for case $\mathrm{A}$ in the lower portion of figure 7 where the data fall on two distinct curves. In a first calibration only values on 
the lower curve were found, but in a second, some weeks later, values on both curves appeared. A general increase in the scatter of case B as $10^{2} \nu \mathrm{T}$ is reduced below about 0.9 , and a tendency for the slope of case A to steepen below this same value, suggest some change in regime at this point, possibly a transition to turbulent flow. At least some of the variation for higher values of $10^{2} \nu T$ might derive from cellular types of flow related to those between concentric cylinders and investigated by G. I. Taylor. But however interpreted, the scatter in figure 7 demonstrates the failure of a viscous manometer of the present design.

It must also be noted, that because any change in the pattern of flow in the cavity requires the action of viscous forces, the rotor response of this type of manometer is relatively slow. In the range of the smaller pressure differences, the establishment of a near steady state required from one to several minutes.

There may be other designs for a viscous manometer which would prove more successful. In the present case a reduction of the gap might reduce the scatter to an acceptable degree. Alternative designs of simple geometry and construction are readily conceived but these are not investigated here.

\section{Manometer Sensitivity}

\subsection{Effect of Circuit Resistance and Test Section Design}

If the pressure $\Delta p$ is not precisely adjusted to $\Delta p^{\prime}$, the imbalance, say $\delta p$, is accompanied by a volume rate of flow through the system, say $\delta q$, and in the illuminated cross section, say of area $A$, there is an average velocity of flow $\delta v$ equal to $\delta q / A$.

Taking $\delta v$ to be inversely proportional to the time, $t$, that a particle remains within the two wire lines shown in figure 2, and determining $\delta p$ by either of the two calibration systems, it was found that $\delta v$ increased, approximately, in proportion to $\delta p$ for any given combination of manometer and calibration system. Further investigation, with the centrifugal manometer, confirmed that $\delta v / \delta p$ remained unaffected by $\Omega$.

Thus the flow through the system due to the pressure imbalance can be taken as laminar, and

$$
\frac{1}{\mu} \frac{\delta p}{\delta v}=A R
$$

where $R$ defines the resistance of the system to the flow $\delta q$, through it. Since the smallest pressure which can be measured depends upon the smallest velocity which can be detected, the sensitivity of a manometer system increases with $\delta v / \delta p$ and, to maximize sensitivity, $A R$ must be minimized.

The design of the conduit in the vicinity of the illuminated particles, the test section, determines $A$ and greatly affects the sensitivity of a manometer system. Denoting by $r$ that part of the total resistance $R$ which is affected by the test section design, and by $r_{0}$ the remainder, which may be assumed irreducible, there is an optimum design which minimizes $A\left(r+r_{0}\right)$. Any change in $r_{0}$, as by replacing one pitot-static tube with another, requires a new optimum design. In these investigations, different values of $r_{0}$ correspond to different combinations of manometer and calibration system.

One practical approach to an optimum variable detector design is to vary the depth of the test section. In the case tested here, the glass walls of the test section were plane and parallel and fixed at $1 / 16$ in. apart. Between the walls were fitted a fixed roof and a moveable floor of brass. Both were curved to give a minimum cross section at the plane of illumination. These features are indicated in figure 2. Moving the floor up or down changed both $A$ and $r$ and, consequently, the sensitivity.

Figure 8 shows the observed variation of the sensitivity with depth of test section, for three values of $r_{0}$. The ordinate, $t \delta p / \mu$, is approximately proportional to $\delta p / \mu \delta v$, that is, inversely proportional to the sensitivity. The abscissa, $z$, is the depth of the test section. The solid lines $(1,2,3)$ represent $A\left(r+r_{0}\right)$ with values of $r_{0}$ in the ratios $1: 6: 13$ and with $r$ a function of $z$ chosen best to fit the data. While this fit is not very close, the data show that, with increasing $r_{0}$, the sensitivity decreases and the value of $z$ for "optimum design" also decreases.

The test section depth was not made adjustable until after the manometer calibrations were completed. During the calibrations it was constant at $0.0625 \mathrm{in}$. (equal to the width of the section), a value selected from a preliminary calculation. As seen in figure 8, varying degrees of sensitivity were thereby sacrificed.

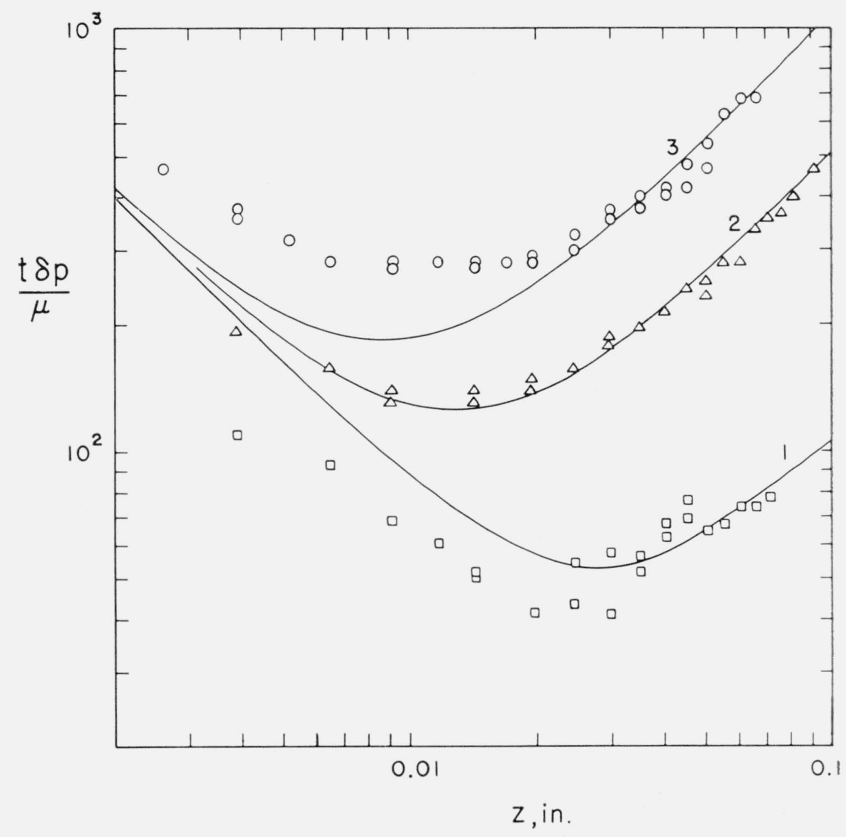

Figure 8. Variation of sensitivity with depth of test section $\mathrm{z}$, and resistance to flow, $\mathrm{r}_{0}$.

On the curves $(1,2,3)$ values of $r_{0}$ are in the ratio 1:6:13. 
It is noted that the resistance of any element in the circuit can be determined easily by connecting the ends to open vessels of water of differing head and measuring the volume flow in a given time.

\subsection{Drift Errors and Corrections}

Experience showed that the factor limiting the accuracy of the calibrations was not, in general, an inability to detect $\delta v$ but rather drifts through the system caused by variations in temperature. Presumably these drifts arise primarily from temperature induced variations in the density of the water from place to place in the system, and perhaps in part from differing rates of expansion of the solid members of the system. In any case, before calibration, with $\Omega$ and $\Delta p$ equal to zero, any drift observed through the microscope could be eliminated. This was done by adjusting the position of the end of a warm copper rod between the vertical elements of a $U$-shaped section of the conduit. The rod was heated by the light source which was near its other end. However, immediately after a reading with $\Delta p$ again zero, a drift was often observed. This drift implied an error $\delta p$ to be added to or subtracted from $\Delta p$, and whose magnitude was given by the known value of $t \delta p / u$. For values of $\Delta p$ above some limit the drift corrections were inconsequential but became critical as $\Delta p$ was reduced to the average magnitude of $\delta p$. In the case of the centrifugal manometer these limits were roughly 1 dyne $\mathrm{cm}^{-2}$ and $1 / 10$ dyne $\mathrm{cm}^{-2}$. The manometer system was partly insulated from the light source and was ventilated by a fan. No attempt was made to measure the temperature field around the rotor housing except to note that temperature differences were too small to be reliably determined by a thermometer with increments of $0.1^{\circ} \mathrm{C}$. However the addition of the fan was found to reduce the drift corrections markedly and further attempts at temperature control may hold promise.

The probable significance of a drift correction increases with the time required for the reading. Unfortunately, for the manometers tested, there occurred a slight pulsing of the observed particles with each turn of the rotor, presumably due to irregularities in bearing friction combined with the possibility of slip between the cones. As a result, verification of no flow required an observation lasting through several cycles, the period of which increased with decreasing $\Delta p$. This pulsing, requiring longer readings which allow the temperature drift time to develop, was found to be the single factor most damaging to the convenience and accuracy of the centrifugal manometer.

\section{Conclusions}

There are many possible designs for a rotational manometer, that is, a meniscus free instrument which balances an unknown pressure difference against the known action of a rotating element. One design, the centrifugal manometer, which had been previously investigated and tested successfully in air, has been found here to be also suitable for use in water. This type of rotational manometer has several distinct advantages. The calibration is of simple form and, with careful design and construction, can be predicted, that is, no calibration is necessary. The rotor response of the instrument is rapid, and the pressure difference produced by the rotation is independent of the molecular viscosity, so the measurement of temperature is unnecessary. The single alternate design here investigated, the "viscous" manometer, had none of these advantages and, because of variability in the calibrations, proved unsuitable. The flow patterns in the cavity upon which the somewhat variable pressure differences depend are probably complex and dominated by viscous forces. While improvements are possible, it seems apparent that the centrifugal manometer is the most satisfactory of rotational manometers.

Under the conditions of calibration, the centrifugal manometer gave readings repeatable to within about \pm 2 percent for $\Delta p>1$ dyne $\mathrm{cm}^{-2}$ and to about \pm 10 percent for $\Delta p$ around $1 / 10 \mathrm{dyne} \mathrm{cm}^{-2}$. In nearly all the readings for $\Delta p<10$ dynes $\mathrm{cm}^{-2}$ the Poiseuille calibrating apparatus was used. Because the two orifices in the side of the Poiseuille flow tube were small, the resistance of this apparatus was probably larger than that of many pitot-static tubes of likely dimensions and design. Therefore the performance under calibration was reasonably close to that which might be expected under normal operation.

The sensitivity of the centrifugal manometer tested in water compares favorably with that of micromanometers involving a fluid surface and is similar to that attained by most meniscus free manometers used in air. The smallest detectable pressure differences were roughly twice those detectable by Kemp's instrument in air. Comparison of manometers for use in different media by the pressure differences they can detect is somewhat arbitrary. An alternate criterion is the percentage error in measuring, with a pitot-static tube, the velocity $V$ at a given point in a conduit for a given Reynolds number. This error is proportional to the error in the pressure difference $1 / 2 \rho V^{2}$ which, at room temperature, is about three times larger in water than in air.

The sensitivity of a rotational manometer, or the error to which it is liable, depends upon the resistance of the circuit in which it operates. An increase of the resistance of any element decreases the sensitivity. Therefore any anticipation of probable error in actual operation must rest not only upon the results of calibration but must also compare the resistance of the calibrating apparatus with that of the elements which are to replace it. A manometer's sensitivity is also dependent upon the design and dimensions of the test section in the flow detector. Ideally, the optimum design differs for each value of the circuit resistance. One approach to a variable design has been found practical.

(Paper 66C4-112) 